Morton, Friedrich

Die biologischen verhältnisse der vegetation einiger Höhlen im quarnerogebiete 


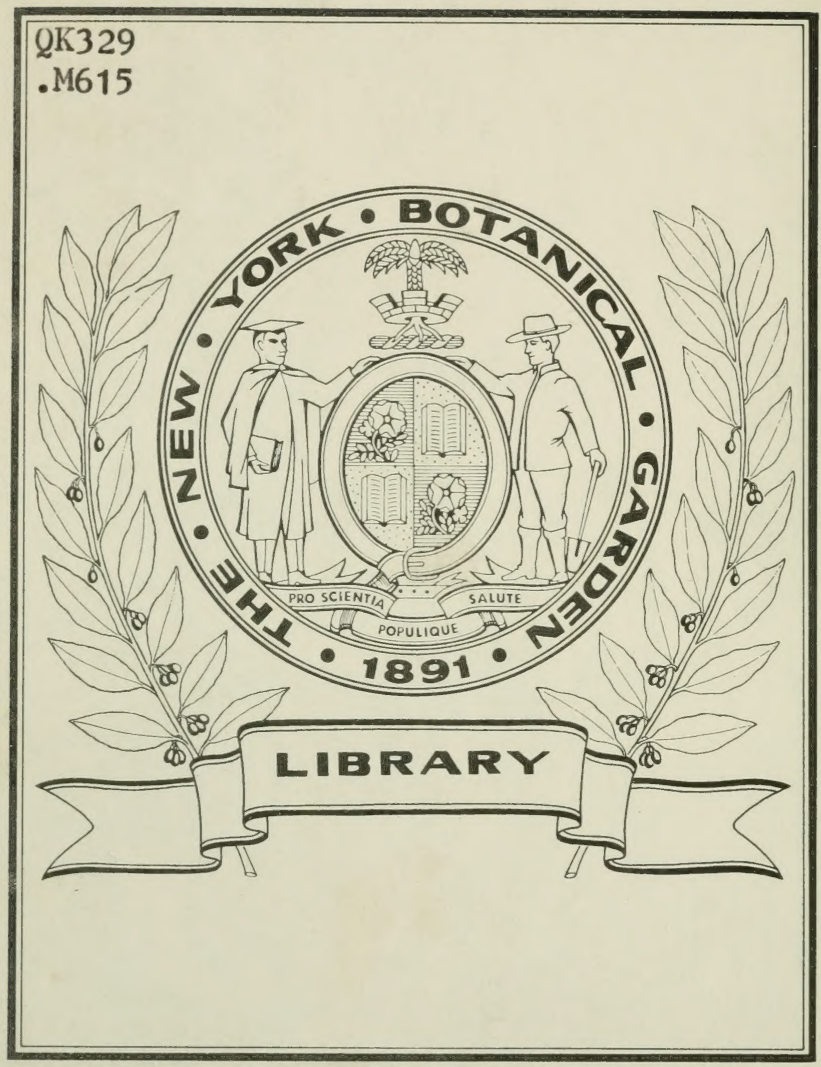






\section{Dio}

biologischen Verhältnisse der Vegetation einiger Höhlen im

\section{Quarnerogebiete.}

Von

\section{Friedrich Morton.}

Separatabdruck aus der „Österreichischen Botanischen Zeitschrift“, Jahrgang 1914, Nr. 7, S. 255-277.

\section{Wien.}

Selbstverlag des Verfassers. - Buchdruckerei Carl Gerold's Sohn. 



\title{
Die biologischen Verhältnisse der Vegetation einiger Höhlen im Quarnerogebiete.
}

\author{
Von Friedrich/Morton (Wien). \\ (Mit 3 Textabbildungen.)
}

Nachdem Wiesners Forschungen den außerordentlichen Einfluß des Lichtes auf die Pflanze dargelegt und die Möglichkeit geboten haben, in exakter Weise die Beziehungen zwischen beiden zum Ausdruck zu bringen, hat sich der Forschung ein großes Arbeitsfeld eröffnet. Die bisher erschienenen Arbeiten haben den großen Einflu\& des Lichtklimas auf die Pflanzenwelt nachgewiesen, so daß heute die Lichtstudien, wenn wir die biologischen Verhältnisse eines Gebietes ganz erfassen wollen, eine hervorragende und unentbehrliche Rolle spielen. Daher finden wir auch schon in einzelnen der neuesten pflanzengeographischen Arbeiten längere Abschnitte der Erforschung des betreffenden Lichtklimas gewidmet ${ }^{1}$ ).

Von der Erkenntnis dieser großen Bedeutung des Lichtes ausgehend, habe ich im Quarnerogebiete mit Lichtstudien begonnen, die meine pflanzengeographischen Studien daselbst in biologischer Richtung hin ergänzen sollen. Davon übergebe ich jetzt einen kleinen Spezialabschnitt der Öffentlichkeit. Die biologischen, speziell die Lichtverhältnisse und ihre Einwirkung auf die grüne Pflanzenwelt der Höhlen waren lange Zeit von der Forschung ganz unbeachtet geblieben, obwohl gerade das abgeschwächte Licht und die unter seinem Einfluß stehende Vegetation manche interessante Beziehungen erwarten lassen konnte. Erst Lämmermayr, ein Schüler Wiesners, wandte sein Augenmerk der grünen Pflanzenwelt der Höhlen zu und legte seine Untersuchungen in einer großen Arbeit ${ }^{2}$ ) nieder, in der ein umfassendes Tatsachenmaterial

1) So z. B. in E. R ü b e l, Pflanzengeographische Monographie des Berninagebietes 1911; J. B r a u n, Die Vegetationsverhältnisse der Schneestufe in den RätischLepontinischen Alpen 1913.

$\left.{ }^{2}\right)$ L. L ä m m e r m a y r, Die grüne Pflanzenwelt der Höhlen. I. Teil, Denkschr. d. math.-naturwiss. Kl. der k. Akad. d. Wiss. Wien, LXXXVII. Bd., 1911 u. 1913. 
zusammengestellt ist und manche wertvolle Beiträge zur Biologie der Höhlenflora zu finden sind.

Dadurch angeregt, entschloß ich mich, die Höhleu in meinem pflanzen geographischen Arbeitsgebiete einer Untersuchung zu unterziehen, wobei mir bei der Durchführung derselben Lämmermayrs Arbeit vorbildlich gewesen ist.

\section{Höhle auf der Punta Ferkanjo (Insel Arbe).}

Diese Höhle liegt in ungefähr $2 \mathrm{~m}$ Seehöhe auf der Ferkanjo ge . nannten Landzunge gegenüber der Stadt Arbe im Kreidekalkstein. Zu dem nach Norden gerichteten Höhleneingang, der ca. 1.25 m unter der Oberfläche liegt und im Maximum $1.4 \mathrm{~m}$ hoch und $2.6 \mathrm{~m}$ breit ist, führt eine kurze Bodeneinsenkung. Von hier fällt der Boden der Höhle rasch weiter um einen halben Meter. Die Tiefe der Höhle beträgt $5 \mathrm{~m}$, ihre durchschnittliche Höhe $1.8 \mathrm{~m}$, jedoch senkt sich die Decke nach den Seiten hin sehr rasch hinab, so daß man sich rechts und links vom Eingang nur kriechend fortbewegen kann. Die Höhle ist außerordentlich feucht (überall Tropfsteinbildung), ihr Boden zum Teil sehr humusreich und von tierischen Exkrementen ${ }^{1}$ ) durchsetzt, zum Teil steinig und felsig, durchwegs aber stark uneben. Unmittelbar am Höhleneingang, dessen Felsen am Vormittag durch viele Stunden direktes Sonnenlicht erhalten, das aber nicht in die Höhle dringt, wachsen bei $\left.\mathrm{L}^{2}\right)=\frac{1}{5 \cdot 5}(8$. VIII. 1913, $3 \mathrm{Uhr}$ nachmittags, diffuses Licht) folgende Arten :

Parietaria judaica L., Silene angustifolia Guss., Aethionema saxatile (L.) R. Br., Viola alba Bess., Pistacia lentiscus L., Sedum acre L., Rubus ulmifolius Schott, Teucrium montanum L., Stachys fragilis Vis., Rubia peregrina L., Helichrysum italicum (Roth) Guss., Reichhardia picroides (L.) Roth, Asparagus acutifolius L., Melica ciliata L., Dactylis hispanica Roth, Brachypodium rupestre (Host) R. et Sch., Agropyron litorale Boiss.

Diese Pflanzen sind durchwegs Elemente der Garrigue und der steinigen Trift, welche Formationen den größten Teil der Landzunge einnehmen. Im Höhleninnern wuchsen 8 Arten.

1. Adiantum capillus Veneris L, reicht bis $4.8 \mathrm{~m}$ Tiefe, dringt also unter den Phanerogamen am weitesten nach innen. Der relative Lichtgenuß beträgt hier $\mathrm{L}=\frac{1}{1 \gamma_{00}}\left(\right.$ ? $\left.^{3}\right)$. Die Pflanzen erwiesen sich als vollkommen euphotometrisch, die Blätter also deutlich in eine zur Richtung des einfallenden diffusen Lichtes senkrechte Ebene gestellt. Da das Licht nahezu parallel dem Höhlenboden einfällt, sind besonders weiter im Höhleninnern die Stengel wagrecht dem Boden anliegend, positiv beliotropisch und die Blätter senkrecht dazu, also fast vertikal gestellt, was einen höchst eigenartigen Anblick bietet. Die letzten fertilen

$\left.{ }^{1}\right)$ In der Höhle hausen auch viele Fledermäuse (große Hufeisennase).

2) Hier und im folgenden bezeichnet $L$ stets den relativen Lichtgenuß.

3) Dieser Wert ist natürlich nur ein A n n̈̈ herung swert; einerseits ist in dieser Tiefe die Zusammensetzung des Lichtes eine geänderte, anderseits können in so dunklen Räumen exakte Messungen nicht mehr durchgeführt werden. Dieser Fall ist ein ganz abnormer, der die außerordeutliche Anpassungsfähigkeit dieses Farnes zeigt, der hier bei einem weit unter seinem Minimum liegenden Lichtgenusse lebt. 
Exemplare traf ich in $3.5 \mathrm{~m}$ Tiefe an. Die aus einer Tiefe von 4 bis $4.8 \mathrm{~m}$ stammenden Pflanzen sehen so abweichend vom Typus aus (Abb. 1), daß ich mich entschloß, dieselben als eigene neue Form zu beschreiben ${ }^{1}$ ), um so mehr, als ich dieselbe Ausbildungsweise auch in mehreren Höhlen am NO-Absturze des Tignarogebirges beobachten konnte. Prof. Paulin schlug für diese Form den Namen f. subintegrum vor;

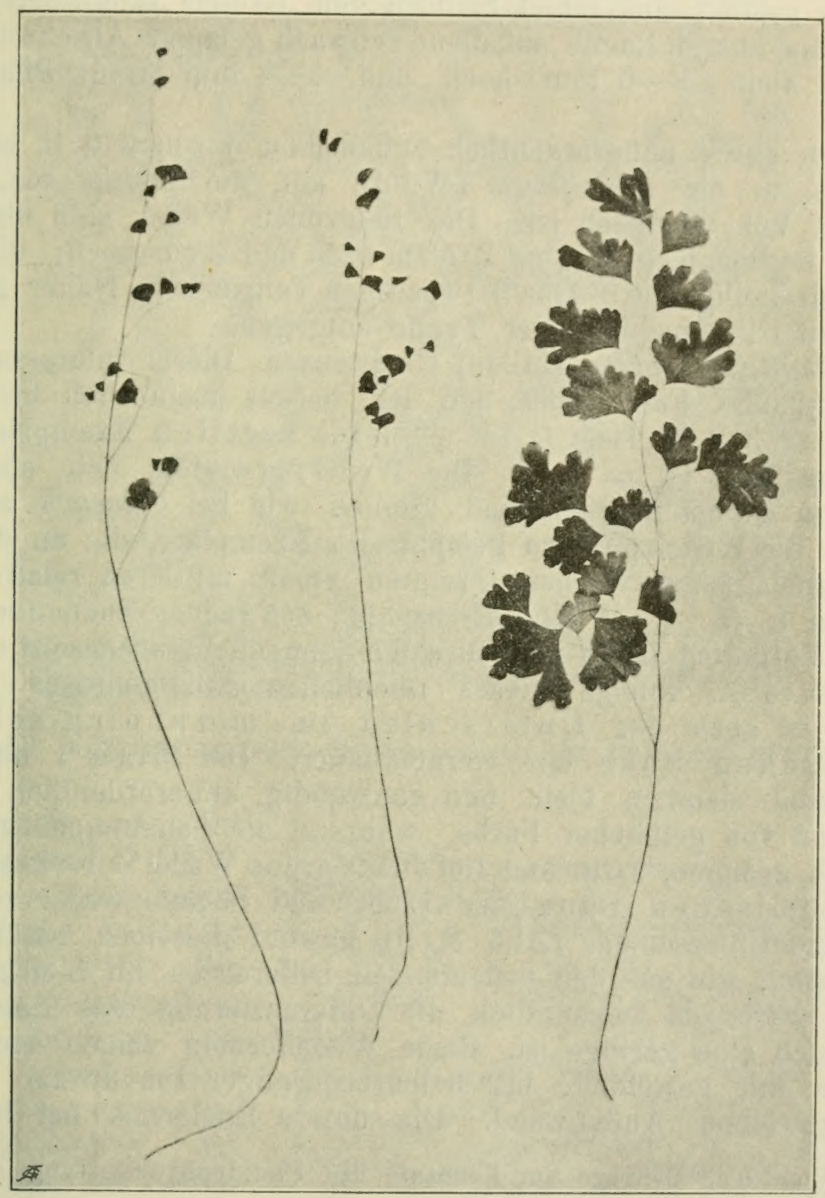

Abb. 1. Adiantum capillus Veneris, forma trifidum (rechts) und forma subintegrum (links).

ich gebe im folgenden eine Beschreibung derselben und erlaube mir Herrn Prof. Paulin als Mitautor anzuführen.

1) Die Aufstellung bezweckt hier nicht eine Belastung der systematischen Nomenklatur; es soll vielmehr damit nur die Vorstellung an einen ganz bestimmten, bi ologisch bedingten Typus verbunden werden und der Name als Terminus bei Sehilderung von Höhlenfloren und ähnl. dienen. 
Adiantum capillus Veneris L. f. subintegrum Morton et Paulin, nova f. Blätter einzeilig, sehr schütter gestellt, außerordentlich zart, $15-20 \mathrm{~cm}$ lang. Stiel bis $25 \mathrm{~cm}$ lang, meist etwas länger als die Spreite. Spreite sehr stark in die Länge gestreckt, sehr schwach eiförmig, 1- bis 2 fach gefiedert, hellgrün. Ansatzstellen der Fiedern erster Ordnung bis $4 \mathrm{~cm}$ voneinander entfernt. Fiedern nicht abwechselnd (Fiederchen abwechselnd), sehr zarì und dünn, dabei lang gestielt. Letzte Abschnitte sehr dünn gestielt, aus schief keilförmigem Grunde rhombisch verkehrt eiförmig, am oberen Rande auffallend schwach gelappt. Abschnitte außerordentlich klein, 2-6 $\mathrm{mm}$ hoch und $4-8 \mathrm{~mm}$ breit. Pflanze stets steril.

Neben dieser außerordentlich auffallenden Form tritt in einer Tiefe von $1-2^{1} / 2 m$ die f. trifidum (Willd.) auf, die mit der var. Visianii Schloss. et Vuk. identisch ist. Die tiefgrünen Wedel sind über $30 \mathrm{~cm}$ hoch, die Segmente groß (bis $3.5 \mathrm{~cm}$ breit und $3 \mathrm{~cm}$ hoch), die meisten sehr tief dreiteilig mit 2- (bis 3-) spaltigen Teilstücken. Näher gegen den Eingang zu tritt uns dann der Typus entgegen.

2. Phyllitis hybrida (Milde) Christensen. Dieser interessante, sehr anpassungsfähige Farn, über den ich bereits ausführlich berichtete ${ }^{1}$ ), reicht bis zu $3.9 \mathrm{~m}$ 'Tiefe $\left(\mathrm{L}=\frac{1}{33_{1}}\right)$. Die fertilen Exemplare reichen bis zu $3 \mathrm{~m}$ Tiefe $\left(\mathrm{L}=\frac{1}{98 \cdot 5}\right)$. Die Wedel erwiesen sich ebenfalls als vollkommen euphotometrisch und ähnlich wie bei Ceterach sehr stark bis gegen die Wedelspitze zu gelappt. Die Exemplare, die an den Felsen des Höhleneinganges wuchsen, zeigten einen mittleren relativen Lichtgenußs von $\mathrm{L}=\frac{1}{3.35}\left(4 \mathrm{Uhr} 45\right.$ nachm., schwaches Sonnenlicht $\left.{ }^{2}\right)$, sind aber am Vormittag lange dem direkten Sounenlicht ausgesetzt und zeigen dann $\mathrm{L}=\frac{1}{1 \cdot 36}$. Infolge dieses reichlichen Lichtgenusses gegenüber $\mathrm{L}=\frac{1}{331}$ ist auch der Unterschied im morphologischen und anatomischen Baue ein beträchtlicher. Die Blätter ${ }^{3}$ ) der Sonnenformen sind ziemlich klein und ganzrandig, außerordentlich derb, fast lederig und von gelblicher Farbe, während die Schattenpflanzen große, sehr stark gelappte, zarte und tief dunkelgrüne $\mathrm{Wedel}^{3}$ ) besitzen ${ }^{4}$ ). Auch im anatomischen Baue der Licht- und Schattenwedel zeigen sich augenfällige Unterschiede (Abb. 2,3), obwohl dieselben natürlich nicht so groß sind, wie aus den bedeutenden Differenzen im Lichtgenusse zu erwarten wäre, da bekanntlich die Differenzierung des Mesophylls an und für sich eine geringe ist. Beide Wedelformen zeigen eine kräftige Epidermis mit gewölbter, fast halbkreisförmiger Innenwand und etwas flacher gewölbter Außenwand. Die untere Epidermis hat im wesent-

1) Morton, Beiträge zur Kenntnis der Pteridophytengattung Phyllitis, in Ö. B. Z., 1914, Heft $1 / 2$.

2) Die Eingangsfelsen werden zum Teil am Nachmittag von der Sonne gestreift.

3) Siehe die Abbildung in meiner früher genannten Arbeit.

4) Die von Harač ić unterschiedenen Formen, bzw. Varietäten [f. typica Haračić, f. lobata und f. Reichhardtii (Haračić)] kommen alle in dieser Höhle vor. Ich sehe jedoch von einer Aufzähluug derselben im Zusammenhang mit der Angabe bestimmter Lichtgenußwerte ab, da die von $\mathrm{H}$ a r a č i ć unterschiedenen Formen zwar zum Teil verschiedenen Standort'sverhältnissen entsprechen, aber doch nicht streng auf distinkte biologische Typen aufgebaut wurden. Wollte man überhaupt die verschiedenen Formen mit Namen belegen, wäre es am zweckmäßigsten, nur die typischen Schatten- und Sonnenpflanzen als Formen aufzufassen und die (durch verschiedene Standortsvarianten bedingten) zahllosen Übergänge unbezeichnet zu lassen. 
lichen denselben Bau, nur sind ihre Innenwände vicht so stark nach einwärts gewölbt. Die Zellen der unteren Epidermis enthalten bei der Sonnenform im Gegensatz zur Schattenform kein Chlorophyll, sondern sind mit einer äußerst feinkörnigen Substanz erfüllt, deren chemische

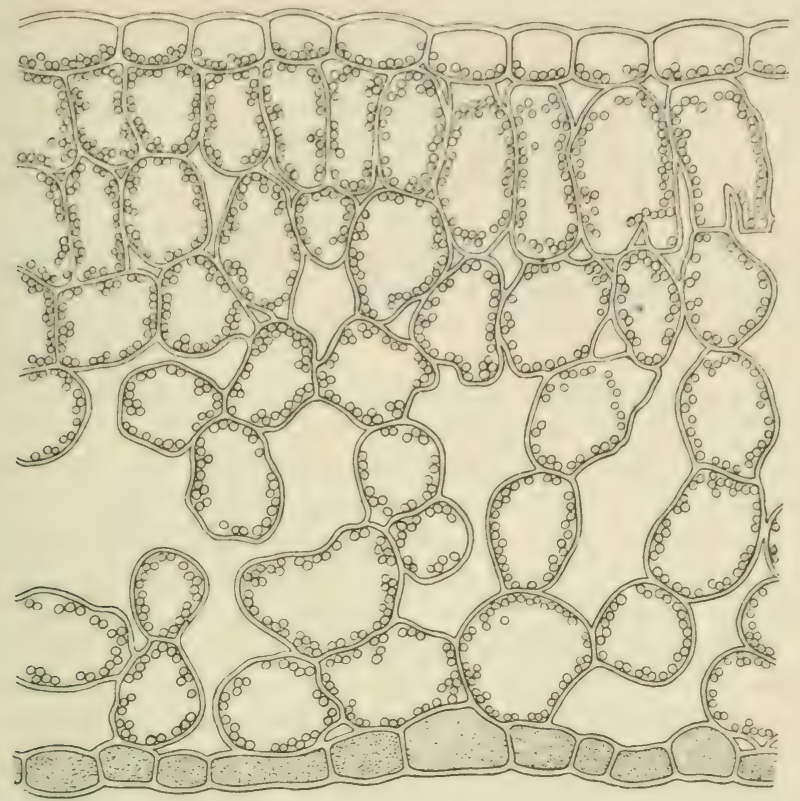

Abb. 2. Wedelquerschnitt der Sonnenform von Phyllitis hybrida.

Beschaffenheit noch nicht ermittelt wurde. Die Hauptunterschiede liegen nun in der Ausbildung des Mesophylls. Die Wedel der Schattenform (Abb. 3) besitzen ein typisches, nicht weiter differenziertes (ungegliedertes) Mesophyll, das nur aus drei bis höchstens vier Zellagen besteht und außerordentlich große Interzellularen aufweist. Auffallend sind die langen schlauchförmigen Zellen

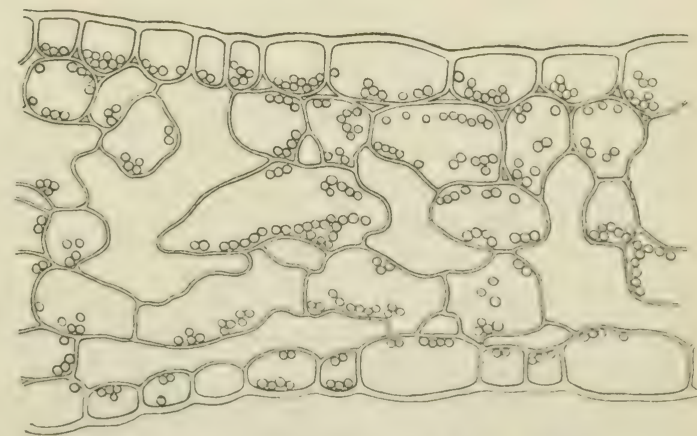

Abb. 3 Wedelquerschnitt der Schattenform von Phyllitis hybrida.

in der untersten Mesophyllschichte. deren starke flächige Verbreiterung jedenfalls im Sinue einer Vergrößerung der assimilatorischen Fläche zu deuten ist. Die Zellen der beiden oberen Lagen sind unregelmäßsig polygonal. 
Ganz anders sieht nun das Mesophyll bei den Sonnenwedeln (Abb. 2) aus. Es besteht im Durchschnitte aus 5-7 Zellreihen und zeigt deutliche Ansätze zu einer Differenzierung in ein Palisaden- und Schwammparenchym. Die erhöhte Lichtintensität hat zur Steigerung der Assimilationsenergie geführt, was in einer Verstärkung des Mesophylls zum Ausdrucke gekommen ist. Das Palisadengewebe besteht aus $\mathrm{zwei}$ bis drei Lagen im Querschnitte länglich rechteckig erscheinender und ziemlich lückenlos aneinanderschließender Zellen; diese Reduktion der Luftlücken steht mit der enormen Lichtintensität, der diese Sonnenpflanzen ausgesetzt sind, im Zusammenhange und ist auch von anderen Farnen sonniger Standorte (z. B. Botrychium) bekannt. An das Palisadengewebe schlie日t sich ein aus zwei bis vier Lagen bestehendes Schwammparenchym an: das Gefüge ist hier ein viel festeres als bei den Schattenwedeln, die Zellen sind meist kugelig, die flächigen Verbreiterungen fehlen vollkommen.

Die Querschnittsdicke der Wedel der Schattenform verhält sich zu jener der Lichtform so wie $2 \cdot 1: 1$.

Diese Ausführungen zeigen, daß im Einklang mit dem anatomischen Wedelbaue anderer Farne mit ausgeprägten Licht- und Schattenformen auch hier Ansätze zu einer dorsiventralen Entwicklung vorhanden sind, die vor allem in der Ausbildungsweise des Mesophylls zum Ausdrucke kommen. Diese Tatsache ist deshalb von besonderem Interesse, weil, wie die Untersuchungen Russows an Marsilia quadrifolia gezeigt haben, bei den entwicklungsgeschichtlich höherstehenden heterosporen Pteridophyten (weuigstens zum Teil) diese Trennung des Mesophylls in ein Palisaden- und Schwammparenchym schon scharf zur Durchführung gekommen ist.

Wie ich in meiner früher zitierten Arbeit ausgeführt habe, ist Phyllitis hybrida eine ausgesprochene Schattenpflanze, die Höhlen und Felsspalten bevorzugt. Dieser Charakter kommt auch im anatomischen Bau unverkennbar zum Ausdrucke, wofür vor allem die deutliche Tendenz zur Vergrößerung der transpirierenden Oberfläche (Ausbildung der langgestreckten Mesophyllzelleu bei den extremen Schattenwedeln!) des Schwammgewebes und das stark gelockerte, oft überhaupt nicht $\mathrm{zu}$ unterscheidende Palisadengewebe sprechen. Die starken Abweichungen bei den Sonneriwedeln stehen mit der überhaupt großen Anpassungsfähigkeit dieses Farnes im Zusammenhang.

3. Asplenium trichomanes L. geht bis in $3.8 \mathrm{~m}$ Tiefe; die fertilen Exemplare reichen nur bis ca. $2 \cdot 25 \mathrm{~m}$. Die Wedel erwiesen sich als vollkommen euphotometrisch. Neben Exemplaren, die zur f. typicum Luerssen gehören und sich nur durch etwas größere dunkelgrüne Fiedern von Pflanzen lichtreicherer Orte unterscheiden, kommt auch noch die f. lobati-crenatum Lam. et DC. vor; einzelne Pflanzen neigen durch ihre länglichen, grobgekerbten Fiedern mit nur ein bis zwei Paaren von Sori zur f. umbrosum (Luerssen). Überall trifft man zwischen ausgewachsenen Exemplaren Jugendformen, die besonders mit der Tiefe an Zahl zunehmen, stationär zu sein scheinen und sich im Aussehen der vou L ä m me rmayr (1911, p. 10) beschriebenen und abgebildeten Form stark nähern. Auch zahlreiche Vorkeime dieser Art sowie von Adiantum 
capillus Teneris wachsen auf dem Höhlenboden und sind ebenfalls streng euphotometrisch, also vertikal zum Boden orientiert.

4. Parietaria judaica L. geht bis in $2.45 \mathrm{~m}$ 'Tiefe. Blätter ebenfalls vollkommen euphotometrisch.

5. Ceterach officinarum Lam. et DC. geht bis $2 \mathrm{~m}$ Tiefe $\left(\mathrm{L}=\frac{1}{35.4}\right)$. Wedel vollkommen euphotometrisch. Die Spreite ist sehr dünn, die Sprenschuppen schütter. Manche Exemplare neigen durch Segmente mit schwach gekerbten Rändern zur f. crenatum Moore. Daneben finden sich zahlreiche Jugendformen mit ganz unregelmäßigen Segmenten. euphotometrisch.

7. Tamus communis L. und die var. cretica Boiss. gehen bis $0.8 \mathrm{~m}$ Tiefe und erwiesen sich nur als schwach euphotometrisch.

8. Reichhardia picroides (L.) Roth geht bis $0.5 \mathrm{~m}$ Tiefe und zeigt ziemlich euphotometrische Blätter.

Die Hinterwand der Höhle, wo nur mehr sehr stark gedämpftes Licht herrscht. und die Steine am Höhlenboden sind mit einem größtenteils grünen Überzuge ron Algen bedeckt. Ich fand Protococcus viridis Ag., Glococapsa aeruginosa (Carm.) Kütz und Grl. alpina (Näg.) Brand. Von Moosen sammelte ich in einer Tiefe von $4 \mathrm{~m}\left(\mathrm{~L}=\frac{1}{357}\right)$ Cephalosic bicuspidata (L.) Duw.

Die Temperatur betrug (8. VIII. 4 Uhr nachm.) am Höhleneingang im Schatten $20 \cdot 8^{\circ} \mathrm{C}$, im Höhleninnern $19-18 \cdot 7^{\circ} \mathrm{C}$.

\section{Jamina-Höhle (Insel Arbe).}

Diese Höhle liegt am NW-Abhange des Cruna vrh (an der einen Seite der Valle Jamina) im Kreidekalk. Wenn man vom trigonometrischen Höhenpunkte 125 wenige Schritte den NW-Abhang hinabklettert, steht man vor dem Eingange dieser ca. $110 \mathrm{~m}$ über dem Meere liegenden Höhle, der durch zwei mächtige Feigenbäume beschattet wird. Der ungefähr nach Norden gekehrte, torförmige Eingang ist etwas über mannshoch und führt in das tunnelförmige Höhleninnere. Zunächst folgt man einem geraden, ungefähr von Norden nach Süden verlanfenden Gange, der bei einer durchschnittlichen Breite von $2.5-3 \mathrm{~m}$ eine Länge ron ca. $18 \mathrm{~m}$ erreicht; dann biegt er nach SW (ca. 30 $0^{\circ}$ ab, erweitert sich gleichzeitig und geht endlich in eine nach SO und eine nach SW gerichtete Nische über. In diesem erweiterten 'Teile ist die Höhle am höchsten, und zwar bis $4 \mathrm{~m}$ hoch. Sonst bleiben Höhe und Breite sehr konstant. Überall ist Tropfwasser und Tropfsteinbildung. In den dunklereu Partien bängen zahlreiche grole Hufeisennasen. Der Boden ist stark schlüpfrig und mit vielen Exkrementen ron Schafen bedeckt. die hier gerne vor den Gluthitzen des Sommers Schutz suchen. Am Eingang und unmittelbar vor demselben wuchsen bei $\mathrm{L}=\frac{1}{6}$ :

Asplenium trichomanes L., Ceterach officinarum Lam. et D(J., Urtica dioica L., $\mathrm{r}^{1}$ ), Parietaria judaica L., Sisymbrium officinale (L.) Scop., r, Sedum acre L., Rubus ulmifolius Schott. Cynoglossum creticum Mill., r, Cymbalaria muralis G. M. Sch., Scolymus hispanicus L., r,

1) $\mathrm{r}=$ Ruderalpflanze. 
Asparagus acutifolius I., Gastridium lendigerum (L.) Gaud., Dactylis hispanica Roth, Hordeum leporinum Lk., r.

Neben Elementen der steinigen Triften treten also hier mehrere Ruderalpflanzen (davon Urtica in großen Mengen) auf, was mit dem Besuch dieser Lokalität durch Schafe im Zusammenhang steht. Am weitesten in das Innere (sowohl am Höhlenboden als auch an den Seitenwänden bis zu einer Höhe von $2 \mathrm{~m}$ ) reicht hier Asplenium trichomanes L., nämlich bis zu $7 \cdot 5 \mathrm{~m}$ Tiefe $\left(\mathrm{L}=\frac{1}{710}\right.$ ?). Die Wedel sind vollkommen euphotometrisch. Die fertilen Exemplare reichen bis $2.5 \mathrm{~m}$ Tiefe; die Pflanzen aus $7.5 \mathrm{~m}$ Tiefe sind teils sterile, tief dunkelgrüne Pflänzchen, die zur f. umbrosum Milde gehören, teils stationäre Jugendformen.

Außerdem fand ich hier euphotometrische Prothallien von Adiantum capillus Veneris L. Farnprothallien sind sowohl hier als auch in anderen Höhlen an Zahl den ausgewachsenen F'arnpflanzen weit überlegen. Parietaria judaica reicht bis in $2.7 \mathrm{~m}$ Tiefe, Ceterach bis $1.8 \mathrm{~m}\left(\mathrm{~L}=\frac{1}{35}\right)$; beide Pflanzen besitzen vollkommen euphotometrische Assimilatiousorgane. Bei $1.5 \mathrm{~m}$ fand ich Vorkeime von Asplenium ruta muraria L. $\left(\mathrm{L}=\frac{1}{28 \cdot 6}\right)$.

$\nabla_{0 \text { n }}^{2 \cdot 6}$ Moosen sammelte ich bis zu $7.5 \mathrm{~m}$ Tiefe am Boden und an den Wänden Rhynchostegiellı algiriana (Brid.) Broth. (fertil bis $3 \mathrm{~m}$ Tiefe) und bei $1.5 \mathrm{~m}$ sterile Stengel von Tortella flavovirens (Br.) Broth.

Der Boden, sowie die Höhlenwände, zum Teil sogar die Decke, sind von einem grünen Überzuge bedeckt, der bis an die hintere Höhlenwand reicht, also bis in eine Tiefe von $23 \mathrm{~m}$ ( $L=\frac{1}{1500}$ ? ?). Er wird ausschließlich von Protococcus viridis Ag. und seiner f. minor gebildet.

Die Temperatur betrug am 7. VIII., um 3 Uhr nachm., im Schatten am Höhleneingang $+19^{\circ} \mathrm{C}$, bei $3 \mathrm{~m}$ Tiefe $+17 \cdot 2^{\circ} \mathrm{C}$, bei $23 \mathrm{~m}$ $+16 \cdot 3^{\circ} \mathrm{C}$.

\section{Vela jama (,grande grotta66) auf der Insel Lussin.}

Diese schöne Höhle befindet sich am SW-Abhange des Mte. Ossero auf der Insel Lussin in einer Höhe von $450 \mathrm{~m}$ über dem Meeresspiegel. Sie liegt im Kalkstein ungefähr unter dem Hauptgipfel des Berges (Televrina), im Bereiche der mächtigen Felswände, die in halber Berghöhe längs der ganzen Westseite desselben in einer Länge von $3.5 \mathrm{~km}$ laufen und eine ehemalige Meeresküste mit prachtvollen (hohlkehlenartig ausgehöhlten) Brandungsreliefs darstellen. Die Höhlenöffnung wird durch ein ungeheures, annähernd dreieckiges Portal gebildet, das ca. $8 \mathrm{~m}$ hoch und $5 \mathrm{~m}$ breit ist und sich nach $\mathrm{SW}$ öfnet. Im Innern zeigt die $25 \mathrm{~m}$ lange Höhle, dis sich nach oben gotisch zuspitzt, einen dreieckigen Querschnitt. Sie verläuft in einem leicht geschwungenen Bogen und wird gegen das Innere zu immer niedriger und schmäler, bis sie schließlich mit einer Breite von $0.5 \mathrm{~m}$ und einer Höhe von kaum $1 \mathrm{~m}$ endet. Die Höhle ist ganz trocken, der Boden eben und mit tierischen Exkrementen bedeckt, die zum großen Teile ebenso wie bei den vorher besprochenen Höhlen von Schafen berrühren.

An den Felsen des Eingangsportales notierte ich bei $L=\frac{1}{1 \cdot 1}$ folgende Pflanzen: 
Parietaria judaica L., Euphorbia Wulfenii Hoppe. Silene angustifolia Giuss., C'lematis flammula L., Alyssum medium Host, Biscutella cichoriifolia Loss., P'runus mahaleb L., Melilotus officinulis (L.) Lam., r, Coromilla emeroides Boiss. at Sprun., Hedera hadix L., Cymbalaric muralis (i. M. Sch.. Tencrium montunum L., Murrubium candidissimum L., Sintria officinalis L., Origanum hirtum Lk., C'ephalaria leucanthu (L.) Schrad.. Helichrysum italicum (Roth) Guss., Reichthardia picroides (L.) Roth. Gastridium lendigerum (L.) (iaud., Sesleria intermeta Vis.

Am weitesten nach innen reicht Purietaric julaicu. und zwar bis z11 $15 \mathrm{~m}$ Tiefe $\left(\mathrm{I}_{2}=\frac{1}{19 \cdot 9}\right)$ Dann folgt Asplenium trichomanes $\mathrm{L}$. bis zu $14 \cdot 7 \mathrm{~m}$ Tiefe, und zwar durchwegs in fertilen Exemplaren. Asplenium ruta muraria L. reicht bis zu $8 \mathrm{~m}$ 'Tiefe. Cymbalaria muralis, Ruliu peregrina L. und Rubus ulmifolius Schott gehen bis $7 \mathrm{~m}$, Alyssum medium bis $3 \mathrm{~m}\left(\mathrm{~L}=\frac{1}{1.69}\right)$.

Von Moosen fand ich Eucladium styriacum Glow.. das bis $7 \mathrm{~m}$ hineinreicht und sich anch an den Seitenwänden und der Decke findet.

Auch in dieser Höhle sind die Gesteinstrümmer am Boden. Decke und Seitenwände mit grüuen Algen überzogen. Die Hauptrolle spielt wieder Protococcus viridis Ag., der bis an die hintere Höhlenwand $(\mathrm{L}=i$ ) reicht. Hier an dieser hinteren Höhlenwand, also in $20 \mathrm{~m}$ 'Tiefe, fand ich auch Aphanocapsa cinerea Lemm.-nora sp.') und Plectonemu nostocurum Gom., die auch sonst in der Höhle verbreitet, wenn auch nicht so häufig sind wie Protococcus. Bei $6 \mathrm{~m}$ Tiefe wurde Tolypothrix calcarea Schmidle gesammelt.

Die Lichtmengen nahmen in folgender Weise ab: Am Höhleneingang betrug $\mathrm{L}=1$, bei $1 \mathrm{~m}$ Tiefe,$\frac{1}{1.56}$. bei $2 \mathrm{~m} \frac{1}{1 \cdot 64}$. bei $3 \mathrm{~m} \frac{1}{1 \cdot 6 \cdot}$. bei $5 \mathrm{~m} \frac{1}{1 \cdot 86}$, bei $10 \mathrm{~m} \frac{1}{5 \cdot 89}$, bei $15 \mathrm{~m} \frac{1}{19 \cdot 91}$, bei $20 \mathrm{~cm} \frac{1}{5 \cdot 1 \cdot 18}$.

Die Temperaturen betrugen am 17. VIII., 11 Uhr 45 Min. rormittags. am Eingang im Schatten $+20 \cdot 1^{\circ} \mathrm{C}$, bei $1 \mathrm{~m}$ Tiefe $19^{\circ} \mathrm{C}$. bei $2 \mathrm{~m} 18 \cdot 9^{\circ} \mathrm{C}$, bei $5 \mathrm{~m} 18 \cdot 2^{\circ} \mathrm{C}$, bei $10 \mathrm{~m} 174^{\circ} \mathrm{C}$, bei $20 \mathrm{~m}$ $15 \cdot 9^{\circ} \mathrm{C}$.

\section{Organac-Höhle (Insel Lussin).}

Diese Höhle ist de größte auf dem Ite. Ussero. Sie liegt an der SW-Seite in einer Höhe ron lingefähr $300 \mathrm{~m}$ zwischen den beiden Gipfelı T'elevrina und S. Nicolò über dem „na Pećine" genannten Tälehen. Das Gestein ist. Kalk. Ihre Hauptlänge beträgt ca. $40 \mathrm{~m}$; ihr Inneres zerfält in drei Abteilungen, deren letzte ganz finster und schwer zugänglich ist. I) Höhle steht durch drei Öfnungen mit der Außenwelt in Verbindung. Untersucht wurde um die an den einen Enngang direkt anschließende Höhlung. Von der Öffnung tührt ein $1.2 \mathrm{~m}$ hoher und $1.7 \mathrm{~m}$ breiter Gang. der durch dichten Pflanzenwuchs, vor allem durch die wuchernde Crtica und Parietaria stark beschattet wird, nach $2.7 \mathrm{~m}$ Lü̈ge in einen kuppelförmigen Raum ron $2 \cdot 6$ m Breite und $4 \cdot 2$ m Tiefe. An der rechten Seite steht diese Höhle mit den weiteren Teilen des Höhlensystems in Verbindung, erhält ihr Licht aber nur von dem erwähnten Eingange aus. Am Eingange wuchsen bei $\mathrm{L}=\frac{1}{1 \cdot 3}$ folgende Arteu:

1) Diese Alge wurde ron $\mathrm{L}$ e m merm a n $\mathrm{n}$ aus dieser Höhle nen beschrieben und wird nebst den anderen von mir gesammelten Arten von ihm demnärhst publiziert werden. 
Asplenium trichomanes L., Ceterach officinarum Lam. et DC., Urtica dioica L., r, Parietaria julaica L., Euphorbia Wulfenii Hoppe, Acer monspessulanum L., Rubus ulmifolius Schott, Prunus mahaleb L., Coronilla emeroides Boiss. et Sprun., Hedera helix L., Rubia peregrina L., Campanula pyramidalis L., Helichrysum italicum (Roth) Guss.

Bis an die hintere Höhlenwand, also bis in eine Tiefe von $7 \mathrm{~m}$ reicht Asplenium trichomanes ( $\mathrm{L}=\frac{1}{187}$ ). Dann folgt Parietaria judaica bis $6 \mathrm{~m}$, Rubia peregrina bis $5.6 \mathrm{~m}$, Rubus ulmifolius bis $5 \mathrm{~m}$ und Urtica dioica bis $2 \mathrm{~m}\left(\mathrm{~L}={ }_{2.64}^{1}\right)$. Alle Pflanzen besitzen euphotometrische Blätter.

Vou Moosen reicht bis $7 \mathrm{~m}$ Rhynchostegiella algiriana (Brid.) Broth, das hier nur in sterilen Exemplaren gefunden wurde; bei $5 \mathrm{~m}$ Tiefe sammelte ich außerdem Fissidens bryoides Hedw.

Auch hier sind die Gesteinstrümmer am Boden und die Höhlenwände mit dem grünen Überzug des Protococcus viridis Ag. bedeckt, der bis $7 \mathrm{~m}$ Tiefe reicht. Daneben sammelte ich hier an der hinteren Höhlenwand ( $\mathrm{L}=\frac{1}{18 \mathrm{i}}$ ) auch Schizothrix calcicola (Ay.) Gom.

Die Temperatur betrug am Höhleneingang im Schatten (17. VIII., $9 \mathrm{Uhr}$ vorm.) $+17 \cdot 2^{0} \mathrm{C}$, in $1 \mathrm{~m}$ Tiefe $16 \cdot 3^{0} \mathrm{C}$, in $3 \mathrm{~m}$ Tiefe $15 \cdot 6^{\circ} \mathrm{C}$, in $7 \mathrm{~m}$ Tiefe nur mehr $14 \cdot 9^{\circ} \mathrm{C}$. Die Differenz zwischen der Eingangstemperatur und jener bei $7 \mathrm{~m}$ Tiefe betrug also $2 \cdot 3^{\circ} \mathrm{C}$.

Es ist mir noch eine angenehme Pflicht. am Schlusse allen jenen Herre $\mathrm{zu}$ danken, die diese Arbeit durch ibre Mithilfe unterstützten. Herr Gymnasialdirektor Julius Głowacki in Graz hatte die Freundlichkeit, die Moose zu bestimmen, während Herr Dr. Ernst Lemmermann in Bremen die Bestimmung der Algen durchführte. Herrn Professor Alfons Paulin in Laibach verdanke ich die Revision der Farnpflanzen. Schließlich bin ich noch Herrn Hofrat Dr. Julius R. v. Wiesner zu großem Danke verpflichtet, der der Arbeit ein großes Interesse entgegenbrachte und sie durch manche Ratschläge förderte. 


